\title{
Eicosapentaenoic acid attenuates hepatic accumulation of cholesterol esters but aggravates liver injury and inflammation in mice fed a cholate-supplemented high-fat diet
}

\author{
Shiro Watanabe ${ }^{1}$ and Koichi Tsuneyama ${ }^{2}$ \\ ${ }^{1}$ Division of Nutritional Biochemistry, Institute of Natural Medicine, University of Toyama, \\ 2630 Sugitani, Toyama, 930-0194, Japan \\ ${ }^{2}$ Department of Molecular Pathology, Graduate School of Medicine and Pharmaceutical Sciences, \\ University of Toyama, 2630 Sugitani, Toyama, 930-0194, Japan
}

(Received February 21, 2013; Accepted March 7, 2013)

\begin{abstract}
The administration of a sodium cholate-supplemented high-fat (CAHF) diet in mice induced the predominant accumulation of cholesterol esters (CE) in the liver and biochemical and histological features of liver injury. Cholesteryl oleate was the most abundant $\mathrm{CE}$ found in the liver of the mice fed the CAHF diet. We examined the effect of ethyl eicosapentaenoate (EPA) on hepatic CE accumulation and liver injury in the mice fed the CAHF diet. The EPA supplementation suppressed the elevation in the level of cholesteryl oleate in the liver. The expression levels of sterol $O$-acyltransferase- 2 and stearoyl-CoA desaturase-1 mRNA in the liver were elevated in the mice fed the CAHF diet, but they were normalized by the EPA supplementation. However, the elevation in serum transaminase activity, the sign of inflammatory cell exudation and inflammatory gene responses in the liver of the mice fed the EPA-supplemented diet were enhanced compared with those of the mice fed the CAHF diet. We demonstrated that EPA supplementation attenuated $\mathrm{CE}$ accumulation but aggravated liver injury and liver inflammation in the mice fed the CAHF diet.
\end{abstract}

Key words: Bile acid, Non-alcoholic fatty liver disease, Free cholesterol, Cholesteryl oleate, Monounsaturated fatty acid, Polyunsaturated fatty acid

\section{INTRODUCTION}

Bile acids are synthesized from cholesterol in the liver and secreted into the lumen of the small intestine with bile. They play an important role in the digestion and absorption of dietary fats by facilitating the hydrolysis of these fats by pancreatic lipase. In addition to their role in the digestion of dietary fats, bile acids regulate lipid metabolism mainly through nuclear receptors such as the farnesoid X receptor (FXR) (Keitel et al., 2008; Lefebvre et al., 2009). Bile acids profoundly affect cholesterol homeostasis: the dietary supplementation of primary bile acids such as cholic acid or chenodeoxycholic acid elevates cholesterol levels in the blood and liver of experimental animals (Chen et al., 2005; Uchida et al., 1980). These bile acids can repress cholesterol conversion into bile acids in the liver through the downregulation of the expression of cholesterol $7 \alpha$-hydroxylase (Cyp7a1), a late-limiting enzyme for bile acid biosynthesis, which is mediated by FXR activation (Chiang et al., 2000). The dietary administration of bile acids also suppresses lipogenesis through FXR-dependent processes resulting in the reduction in the level of triglycerides (TG) in the serum and liver (Watanabe et al., 2004). Furthermore, dietary supplementation with bile acids induces pathologies similar to cholestatic liver injury in experimental animals (Rost et al., 2003; Song et al., 2011; Teng and PiquetteMiller, 2007; Wang et al., 2003). The elevation in intrahepatic bile acid concentration is considered to directly injure liver parenchymal cells or the bile duct epithelium (Heuman, 1989) in bile-acid-induced cholestatic liver injury. Furthermore, recent studies showed that bile acids

Correspondence: Shiro Watanabe (E-mail: shirowat@inm.u-toyama.ac.jp) 
can directly induce inflammatory responses in experimental liver injury models (Allen et al., 2011; Vergnes and Phan, 2003). In inflammatory responses in the liver, the important role of a nuclear-receptor-mediated mechanism is also suggested (Trauner et al., 2011).

Our previous work demonstrated that feeding female ICR mice with a cholate-supplemented high-fat (CAHF) diet induced the elevation in the level of cholesterol esters (CE) in the liver without a significant increase in the level of TG or phospholipids (PL) in the liver (Watanabe and Tsuneyama, 2010, 2012). In addition, mice fed the CAHF diet exhibited the biochemical and histological features of fatty liver injury (Watanabe and Tsuneyama, 2010, 2012). Notably, the oleic acid (18:1n-9)-containing CE level was markedly elevated in the liver of mice fed the CAHF diet compared with in the liver of those fed the normal diet (Watanabe and Tsuneyama, 2012). On the other hand, the administration of fish oil or its major n-3 polyunsaturated fatty acids (PUFA) is effective in attenuating hepatic steatosis in experimental animals (Hein et al., 2010; Jung et al., 2011; Kajikawa et al., 2009; Sekiya et al., 2003; Tanaka et al., 2010; Yamazaki et al., 2007). Moreover, n-3 PUFA administration could prevent the hepatic accumulation of TG through the suppression of monounsaturated fatty acids (MUFA) generation (Kajikawa et al., 2009). Furthermore, EPA administra- tion was effective in reducing the cholesterol level in the blood and liver (Sugiyama et al., 2008). Here, we examined whether EPA administration attenuates the hepatic accumulation of MUFA-containing CE and subsequently ameliorates liver injury in mice fed the CAHF diet.

\section{MATERIALS AND METHODS}

\section{Animal and diet protocols}

Eighteen female ICR mice (SLC, Shizuoka, Japan) at $5 \mathrm{wk}$ of age were divided into three groups each composed of six mice. The groups were assigned to the powdered basal diet (CE-2, Nippon Clea, Tokyo Japan) as the normal diet, the cholate-supplemented high-fat diet (CAHF diet) and the CAHF diet supplemented with eicosapentaenoic acid (EPA diet) (Table 1). The CAHF diet was prepared by mixing the powdered diet with $20 \%$ beef tallow powder (Nippon Clea), which contains approximately $40 \%(\mathrm{w} / \mathrm{w})$ of fatty acids, and $0.4 \%(\mathrm{w} / \mathrm{w})$ sodium cholate (Wako Pure Chem., Osaka, Japan). The EPA diet was prepared by adding $2 \%(\mathrm{w} / \mathrm{w})$ ethyl eicosapentaenoate (>98\%, w/w, Tama Biochemical Co., Ltd., Tokyo, Japan) to the CAHF diet. The composition of macronutrients in the test diets was calculated and shown in Table 2. The fatty acid contents of the basal powdered diet and beef tallow powder diets were determined by gas-liquid chro-

Table 1. Compositions of the test diets

\begin{tabular}{lccc}
\hline & & $\mathrm{g} / 100 \mathrm{~g}$ diet \\
\cline { 2 - 4 } Ingredients $(\%, \mathrm{w} / \mathrm{w})$ & Normal diet & CAHF diet & EPA diet \\
\hline Powdered diet & 100 & 79.6 & 77.6 \\
Beef tallow powder & 0 & 20.0 & 20.0 \\
Sodium cholate & 0 & 0.4 & 0.4 \\
Ethyl eicosapentaenoate & 0 & 0 & 2.0 \\
\hline
\end{tabular}

Table 2. Composition of macronutrients in the test diets

\begin{tabular}{lccc}
\hline & & $\mathrm{g} / 100 \mathrm{~g}$ diet & \\
\cline { 2 - 4 } & Normal diet & CAHF diet & 7.0 \\
\hline Moisture $(\mathrm{g})$ & 8.9 & 7.1 & 19.4 \\
Crude protein $(\mathrm{g})$ & 24.9 & 19.9 & 21.7 \\
Crude fat $(\mathrm{g})$ & 4.6 & 19.7 & 3.2 \\
Crude fiber $(\mathrm{g})$ & 4.1 & 3.3 & 5.1 \\
Crude ash (g) & 6.6 & 5.28 & 39.8 \\
Nitrigen-free extracts $(\mathrm{g})$ & 51.0 & 40.8 & 441.6 \\
Total energy (kcal) & 344.9 & 423.6 & \\
\hline
\end{tabular}


EPA aggravates experimental fatty liver injury

Table 3. Fatty acid contents of the test diets

\begin{tabular}{lccc}
\hline & & $\mathrm{g} / 100 \mathrm{~g}$ diet \\
\cline { 2 - 3 } Fatty acid & Normal diet & CAHF diet & EPA diet \\
\hline $16: 0$ & 0.88 & 3.17 & 3.15 \\
$16: 1 \mathrm{n}-9$ & 0 & 0.01 & 0.01 \\
$16: 1 \mathrm{n}-7$ & 0.05 & 0.27 & 0.27 \\
$18: 0$ & 0.09 & 1.12 & 1.12 \\
$18: 1 \mathrm{n}-9$ & 0.93 & 3.70 & 3.68 \\
$18: 1 \mathrm{n}-7$ & 0.07 & 0.19 & 0.19 \\
$18: 2 \mathrm{n}-6$ & 2.06 & 1.91 & 1.87 \\
$18: 3 \mathrm{n}-3$ & 0.15 & 0.12 & 0.11 \\
$20: 5 \mathrm{n}-3$ & 0.08 & 0.06 & 2.06 \\
$22: 6 \mathrm{n}-3$ & 0.04 & 0.03 & 0.03 \\
n-3/n-6 & 0.13 & 0.11 & 1.24 \\
Total & 4.35 & 10.58 & 12.49
\end{tabular}

The fatty acid contents of the test diets shown above were calculated from those of the basal diet, beef tallow powder and eicosapentaenoic acid ethyl ester.

matography as described below. The fatty acid contents of the three test diets were calculated on the basis of the fatty acid contents of the basal diet and the beef tallow, which are shown in Table 3. The normal diet contained only a trace amount of cholesterol, but the CAHF and EPA diets contained $0.26 \%$ (w/w) cholesterol, which was derived mainly from beef tallow powder. The mice were housed individually in plastic cages, and the weight of the diets consumed by each mouse was measured using a specially designed feeder (Roden CAFÉ, Oriental Yeast Co., Ltd., Tokyo, Japan). The mice were maintained on the above diets for 3 weeks.

\section{Sampling of blood and liver}

On the final day of the feeding period, the mice were fasted from $0800 \mathrm{hr}$ to $1300 \mathrm{hr}$ and then anesthetized in a chamber filled with isoflurane gas (5\% in air). Trunk blood was collected from the anesthetized mice by cervical dislocation and then centrifuged to obtain serum, which was stored at $-80^{\circ} \mathrm{C}$ until analysis. Three liver specimens (approximately $0.2 \mathrm{~g}$ each) were harvested from the mice and washed in ice-cold saline. One of them was fixed in $10 \%(\mathrm{w} / \mathrm{v})$ formaldehyde for histopathological evaluation. The other two liver specimens for the analyses of lipid and mRNA were quickly frozen in liquid nitrogen and stored at $-80^{\circ} \mathrm{C}$. The above protocols for this experiment were approved by the Committee for Animal Care and Experiments of University of Toyama (Protocol No. S-2010 INM-3).

\section{Analysis of serum and liver lipids}

Frozen liver specimens were homogenized in a mixture of hexane and 2-propanol $(3: 2, v / v)$, and total lipids were extracted as described by Hara and Radin (1978). Total lipids obtained from the liver were dissolved in a small volume of chloroform and then spotted onto silica gel thin-layer chromatography plates (Merck KGaA, Darmstadt, Germany) to purify CE, TG, free cholesterol, nonesterified fatty acids (NEFA) and total phospholipids (PL) as described previously (Watanabe and Tsuneyama, 2012). Fatty acids in the CE, TAG, NEFA and PL fractions were converted into fatty acid methyl esters (FAME) by treating the silica gel absorbents with $5 \%(\mathrm{w} / \mathrm{w})$ methanolic $\mathrm{HCl}$ in the presence of heptadecanoic acid (17:0) as the internal standard for $2 \mathrm{hr}$ at $100^{\circ} \mathrm{C}$. FAME were extracted with petroleum ether and analyzed by gas-liquid chromatography (GC4000, GL Science, Tokyo, Japan) equipped with a capillary column (Rtx65, $0.25 \mathrm{~mm}$ thick x $30 \mathrm{~m}$ long, Restek Corporation, Bellefonte, PA, USA). Helium was used as the carrier gas at a flow rate of $25 \mathrm{~cm} / \mathrm{sec}$. The injector was heated at $250^{\circ} \mathrm{C}$. FAME were detected using a flame-ionization detector (FID) heated at $300^{\circ} \mathrm{C}$. The weights of the fatty acids in the samples were calculated by comparing their peak areas with that of the internal standard added. Free cholesterol was extracted from the silica gel absorbents by the method of Bligh and Dyer (1959) and dried under nitrogen gas. The free cholesterol content of the residue was measured using a commercial assay kit for blood cholesterol determination (Wako 
a

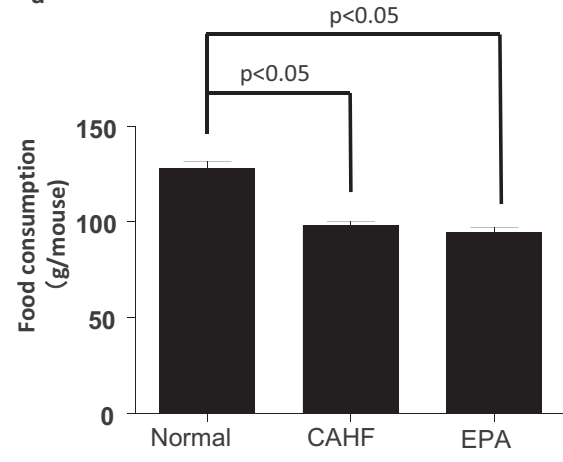

b



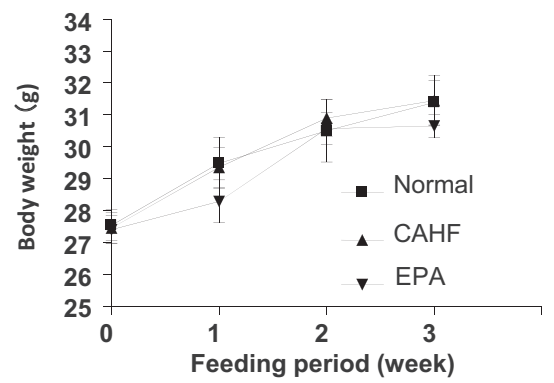

Fig. 1. Food consumption and weight gain of mice. The weight of the diet and total energy ingested by individual mice throughout the feeding period are shown in (a) and (b), respectively. The mice in the CAHF and EPA diet groups ingested significantly smaller $(\mathrm{p}<0.05)$ amounts than those in the normal diet group, although the amounts of total energy intake were not significantly different among the dietary groups. The body weight of mice fed the test diets was measured every week over the feeding period (c), although no significant difference in body weight was observed among the dietary groups.

Pure Chem).

\section{Assessments of liver injury}

Formalin-fixed liver specimens were embedded in paraffin and sectioned for hematoxylin-eosin (HE) staining. The sections were observed under a light microscope. Alanine aminotransferase (ALT) and aspartate aminotransferase (AST) activities in serum were determined using a commercially available assay kit (Transaminase Wako E-test, Wako Pure Chem).

\section{Determination of mRNA expression level in liver}

Total RNA was extracted from the frozen tissues using RNAiso (Takara Bio, Tokyo, Japan) and dissolved in nuclease-free water. RNA concentration was determined by measuring ultraviolet absorption, and a constant amount (100 ng) of total RNA was reverse-transcribed using a PrimeScript kit (Takara Bio) to obtain first-stranded cDNA. After the cDNA was diluted with Easy Dilution (Takara Bio), its aliquot was subjected to a real-time polymerase-chain reaction (PCR) in the presence of SYBR Green (SYBR Green Real-time PCR Master Mix, Toyobo, Osaka, Japan) using a thermal cycler (MyQ2 real-time PCR system, BioRad, Hercules, CA, USA). Sequences of the forward and reverse primers used for the amplification of specific cDNA sequences are listed in Table 4. The amplified products were verified by checking the melting curves after the final cycle of each PCR. The relative levels of cDNA derived from the mRNA of inter- est were expressed as the ratio with respect to that from glyceraldehyde 3-phosphate dehydrogenase (GAPDH) mRNA.

\section{Statistical analysis}

The statistical significance of the difference among the experimental groups was estimated by one-way analysis of variance (ANOVA) followed by a post hoc multiple comparison test with Tukey's method.

\section{RESULTS}

\section{Food intake and body weight gain}

The mice in the CAHF and EPA diet groups ingested significantly less amounts than those in the normal diet groups $(p<0.05)$ during the experiment (Fig. 1a). It was shown that the mice in the three dietary groups have shown similar energy intake (Fig. 1b), which was calculated on the basis of the total energy contents of these diets (Table 2). The mice in the three dietary groups exhibited similar increases in body weight (Fig. 1b).

\section{Fatty acid contents of liver lipid fractions}

The oleic acid (18:1n-9) and palmitoleic acid (16:1n-7) contents were markedly higher in the liver CE of the mice fed the CAHF diet $(4,804 \pm 1,240$ and $654 \pm 252 \mu \mathrm{g} / \mathrm{g}$ liver, respectively) than in the liver $\mathrm{CE}$ of those fed the normal diet $(151 \pm 38$ and $21 \pm 8 \mu \mathrm{g} / \mathrm{g}$ liver, respectively) $(\mathrm{p}<0.05)($ Table 5$)$. The CAHF diet also elevated the 
EPA aggravates experimental fatty liver injury

Table 4. Sequences of primers used for real-time PCR

\begin{tabular}{|c|c|c|c|}
\hline & & & Genebank Acc. No. \\
\hline \multirow[t]{2}{*}{ CCL2 } & Forward & GCATCCACGTGTTGGCTCA & \\
\hline & Reverse & CTCCAGCCTACTCATTGGGATCA & NM_011333.3 \\
\hline \multirow[t]{2}{*}{ CCL 3} & Forward & CATGACACTCTGCAACCAAGTCTTC & \\
\hline & Reverse & GAGCAAAGGCTGCTGGTTTCA & MA095370 \\
\hline \multirow[t]{2}{*}{ CCL 5} & Forward & GGAGTATTTCTACACCAGCAGCAAG & \\
\hline & Reverse & GGCTAGGACTAGAGCAAGCAATGAC & MA131646 \\
\hline \multirow[t]{2}{*}{ Colla1 } & Forward & CTTCTGCAGGGTTCCAACGA & \\
\hline & Reverse & CAGCACCACCAATGTCCAGAG & NM_007743.2 \\
\hline \multirow[t]{2}{*}{ Col3a1 } & Forward & CAGGCCAGTGGCAATGTAAAGA & \\
\hline & Reverse & CTCATTGCCTTGCGTGTTTGATA & NM_009930.2 \\
\hline \multirow[t]{2}{*}{$\mathrm{COX}-2$} & Forward & CTCCTGGAACATGGACTCACTCA & \\
\hline & Reverse & AGGCCTTTGCCACTGCTTGTA & NM_011198.3 \\
\hline \multirow[t]{2}{*}{ Cyp7a1 } & Forward & AGCAACTAAACAACCTGCCAGTACTA & \\
\hline & Reverse & GTCCGGATATTCAAGGATGCA & NM_007824 \\
\hline \multirow[t]{2}{*}{ GAPDH } & Forward & TGTGTCCGTCGTGGATCTGA & \\
\hline & Reverse & TTGCTGTTGAAGTCGCAGGAG & NM_008084.2 \\
\hline \multirow[t]{2}{*}{ HMGCR } & Forward & GCTCTTGTGGAATGCCTTGTGA & \\
\hline & Reverse & TGACATGCAGCCGAAGCAG & NM_008255.2 \\
\hline \multirow[t]{2}{*}{ ICAM-1 } & Forward & AACTGTGGCACCGTGCAGTC & \\
\hline & Reverse & AGGGTGAGGTCCTTGCCTACTTG & NM_010493.2 \\
\hline \multirow[t]{2}{*}{ IL-1 $\beta$} & Forward & TCCAGGATGAGGACATGAGCAC & \\
\hline & Reverse & GAACGTCACACACCAGCAGGTTA & NM_008361.3 \\
\hline \multirow[t]{2}{*}{ IL-6 } & Forward & CCACTTCACAAGTCGGAGGCTTA & \\
\hline & Reverse & GCAAGTGCATCATCGTTGTTCATAC & NM_031168.1 \\
\hline \multirow[t]{2}{*}{ SCD-1 } & Forward & GCCTGTACGGGATCATACTGGTTC & \\
\hline & Reverse & CCAGAGCGCTGGTCATGTAGTAGA & NM_009127.3 \\
\hline \multirow[t]{2}{*}{ SOAT-2 } & Forward & AGACTTGGTGCAATGGACTCGAC & \\
\hline & Reverse & CATAGGGCCCGATCCAACAG & NM_146064. \\
\hline \multirow[t]{2}{*}{ Saa2 } & Forward & GGAACTATGATGCTGCCCAAAG & \\
\hline & Reverse & CACTGCGGCCATGTCTGTT & NM_011314.2 \\
\hline \multirow[t]{2}{*}{ Saa3 } & Forward & GACATGTGGCGAGCCTACTCTG & \\
\hline & Reverse & CTCCATGTCCCGTGAACTTCTG & NM_011315.3 \\
\hline \multirow[t]{2}{*}{ TGF $\beta 1$} & Forward & GTGTGGAGCAACATGTGGAACTCTA & \\
\hline & Reverse & CGCTGAATCGAAAGCCCTGTA & MA122075 \\
\hline \multirow[t]{2}{*}{$\mathrm{TNF} \alpha$} & Forward & AAGCCTGTAGCCCACGTCGTA & \\
\hline & Reverse & GGCACCACTAGTTGGTTGTCTTTG & NM_013693.2 \\
\hline
\end{tabular}

levels of 18:1n-7 (10.1-fold), 16:0 (3.8-fold), and 18:2n-6 (11.1-fold) in the liver CE compared with the normal diet, although these changes were smaller than those in 18:1n9 and 16:1n-7 (> 30-fold). These changes are reflected in the increases in the total fatty acid content of the liver CE of the mice in the CAHF diet group compared with the normal diet group $(7,985 \pm 1,881$ vs. $835 \pm 248 \mu \mathrm{g} / \mathrm{g}$ liver, $\mathrm{p}<0.05)$. In the EPA diet group, the level of 18 : $1 \mathrm{n}-9$ content in the liver CE was much lower than that in the CAHF diet group $(974 \pm 714$ vs. 4,804 $\pm 1,240 \mu \mathrm{g} / \mathrm{g}$ liver, $\mathrm{p}<$ $0.05)$. The levels of other MUFA (16:1n-7 and 18:1n-7) in the liver CE were also lower in the EPA diet group than in the CAHF diet group. The level of 16:0 in the liver CE was lower in the EPA diet group than in the CAHF diet 
Table 5. Fatty acid contents of liver lipid fractions Cholesterol esters

\begin{tabular}{lccc} 
Fatty acid & Normal diet & CAHF diet & EPA diet \\
\hline $16: 0$ & $413 \pm 166^{*}$ & $1,588 \pm 362$ & $624 \pm 348^{*}$ \\
$16: 1 \mathrm{ln} 9$ & $40 \pm 61$ & $60 \pm 41$ & $7 \pm 5$ \\
$16: 1 \mathrm{n} 7$ & $21 \pm 8^{*}$ & $654 \pm 252$ & $99 \pm 88^{*}$ \\
$18: 0$ & $159 \pm 75$ & $326 \pm 121$ & $265 \pm 187$ \\
$18: \ln -9$ & $151 \pm 38^{*}$ & $4,804 \pm 1,240$ & $974 \pm 714^{*}$ \\
$18: 1 \mathrm{n}-7$ & $8 \pm 4^{*}$ & $81 \pm 47$ & $16 \pm 14^{*}$ \\
$18: 2 \mathrm{n}-6$ & $42 \pm 10$ & $470 \pm 112$ & $143 \pm 94$ \\
total & $835 \pm 248^{*}$ & $7,985 \pm 1,881$ & $2,128 \pm 1,313^{*}$
\end{tabular}

\begin{tabular}{|c|c|c|c|}
\hline \multicolumn{4}{|l|}{ Triglycerides } \\
\hline Fatty acid & Normal diet & CAHF diet & EPA diet \\
\hline $16: 0$ & $1,961 \pm 495$ & $1,976 \pm 813$ & $883 \pm 183^{*}$ \\
\hline $16: \ln 9$ & $42 \pm 31$ & $50 \pm 35$ & $22 \pm 13$ \\
\hline $16: 1 \mathrm{n} 7$ & $143 \pm 41$ & $195 \pm 113$ & $44 \pm 30 *$ \\
\hline $18: 0$ & $145 \pm 54$ & $260 \pm 70$ & $222 \pm 124$ \\
\hline $18: 1 n-9$ & $1,399 \pm 424^{*}$ & $3,850 \pm 1,128$ & $1,142 \pm 391^{*}$ \\
\hline $18: 1 n-7$ & $114 \pm 35$ & $167 \pm 62$ & $30 \pm 18^{*}$ \\
\hline $18: 2 n-6$ & $1,508 \pm 437^{*}$ & $880 \pm 327$ & $424 \pm 165^{*}$ \\
\hline total & $5,312 \pm 1,382$ & $7,378 \pm 2,482$ & $2,767 \pm 779 *$ \\
\hline
\end{tabular}

\begin{tabular}{|c|c|c|c|}
\hline \multicolumn{4}{|c|}{ Total phospholipids } \\
\hline Fatty acid & Normal diet & CAHF diet & EPA diet \\
\hline $16: 0$ & $4,570 \pm 656$ & $4,377 \pm 489$ & $4,561 \pm 543$ \\
\hline $16: \ln 9$ & $20 \pm 27$ & $30 \pm 30$ & $16 \pm 13$ \\
\hline $16: 1 \mathrm{n} 7$ & $157 \pm 143$ & $144 \pm 72$ & $79 \pm 12$ \\
\hline $18: 0$ & $2,322 \pm 288$ & $1,900 \pm 199$ & $2,540 \pm 682$ \\
\hline $18: 1 n-9$ & $799 \pm 193^{*}$ & $1,839 \pm 230$ & $1,482 \pm 294$ \\
\hline $18: 1 n-7$ & $239 \pm 82$ & $211 \pm 41$ & $127 \pm 28^{*}$ \\
\hline $18: 2 n-6$ & $2,544 \pm 205$ & $2,425 \pm 230$ & $2,085 \pm 415$ \\
\hline $20: 3 n 6$ & $66 \pm 21^{*}$ & $108 \pm 15$ & $44 \pm 14^{*}$ \\
\hline $20: 4 n 6$ & $1,076 \pm 143^{*}$ & $670 \pm 148$ & $259 \pm 81 *$ \\
\hline $20: 5 n 3$ & $121 \pm 32$ & $92 \pm 51$ & $950 \pm 283^{*}$ \\
\hline $22: 5 n 3$ & $36 \pm 13$ & $21 \pm 14$ & $196 \pm 128^{*}$ \\
\hline $22: 6 n 3$ & $800 \pm 156$ & $871 \pm 316$ & $1,043 \pm 651$ \\
\hline total & $12,749 \pm 928$ & $12,689 \pm 1,339$ & $13,383 \pm 2,879$ \\
\hline
\end{tabular}

Statistically significant difference at $\mathrm{p}<0.05$ compared with the values of the CAHF diet group are marked by *. Fatty acid contents are expressed in $\mu \mathrm{g} / \mathrm{g}$ of liver.

group although its reduction was relatively smaller (1,588 \pm 362 vs. $624 \pm 348$ ) than that in the levels of MUFA in the liver CE. The levels of 18:0 and 18:2n-6 in the liver CE in the EPA diet group were not significantly different from those in the CAHF diet group. The total fatty acid content of the liver CE of the EPA diet group (2,128 $\pm 1,313 \mu \mathrm{g} / \mathrm{g}$ liver) was significantly lower $(-73 \%)$ than that of the CAHF diet group $(7,985 \pm 1,881 \mu \mathrm{g} / \mathrm{g}$ liver $)$ $(\mathrm{p}<0.05)$.

The CAHF diet also induced the elevation in the $18: 1 n-9$ content of the liver TG compared with the nor- mal diet, however, this response was much smaller (2.7-fold) than those in the liver CE ( $>30$-fold). The level of 18:1n-9 in the liver TG in the EPA diet group was lower $(1,142 \pm 391 \mu \mathrm{g} / \mathrm{g}$ liver $)$ than that in the CAHF diet group $(3,850 \pm 248 \mu \mathrm{g} / \mathrm{g}$ liver $)(\mathrm{p}<0.05)$, but similar to that in the normal diet group $(1,399 \pm 424 \mu \mathrm{g} / \mathrm{g}$ liver $)$. In addition, the levels of many types of fatty acid in the liver TG were reduced by the EPA diet, which was reflected by a significant reduction in the total fatty acid contents of this lipid class $(7,378 \pm 2,482$ vs. $2,767 \pm 779 \mu \mathrm{g} / \mathrm{g}$ liver $)$.

There were several fatty acids whose amounts in the 
EPA aggravates experimental fatty liver injury

liver PL were changed significantly by the administration of the CAHF diet: the levels of 18:1n-9 and 20:3n- 6 were significantly elevated but that of $20: 4 n-6$ was reduced. In addition, the levels of the n- 6 series of PUFA (18:2n6, 20:3n-6 and 20:4n-6) were reduced, but those of n-3 PUFA (20:5n-3 and 22:5n-3) were elevated in the liver PL by the administration of the EPA diet. This diet did not change the level of 22:6n-3 in the liver PL. Despite the above changes in the levels of fatty acids in the liver PL, there was no significant difference in the total fatty acid content of the liver PL among the three groups $(12,749 \pm$ $928,12,689 \pm 1,339$ and $13,383 \pm 2,879 \mu \mathrm{g} / \mathrm{g}$ liver in the normal, CAHF and EPA diet groups, respectively).

\section{Expression levels of mRNA of proteins related to cholesterol ester synthesis in liver}

The expression levels of both sterol $O$-acyltransferase- 2 (SOAT-2) and stearoyl-CoA desaturase-1 (SCD-1) mRNA were significantly higher in the liver of the mice fed the CAHF diet than in the liver of those fed the normal diet (Fig. 2) ( $p<0.01$ and $p<0.05$, respectively). The expression level of SOAT-2 mRNA in the EPA diet group was significantly lower than that in the CAHF diet group and not different from that in the normal diet group. The EPA diet also caused a marked reduction in the expression level of SCD-1 mRNA compared with the CAHF diet group. The expression level of Cyp7a1 mRNA was much lower in the liver of the mice fed the CAHF diet than in the liver of those fed the normal diet $(p<0.001)$. However, EPA administration had no significant effect on the expression level of Cyp7a1 mRNA compared with the CAHF diet administration. The CAHF diet also reduced the expression levels of 3-hydroxymethylglutaryl-CoA reduct- ase (HMGCR) mRNA compared with the normal diet $(\mathrm{p}<0.05)$, although no further change was induced by the EPA diet. The expression levels of low-density lipoprotein receptor (LDLR) mRNA were not significantly different among the three dietary groups.

\section{Assessment of liver injury}

As shown, the lipid abnormalities induced by the CAHF diet were efficiently attenuated by the addition of EPA to the CAHF diet, which was reflected in the changes in liver appearance (Fig. 3). Unlike that of the normal diet group, the liver of the CAHF diet group had a white tinge and enlarged (Fig. 3a). The weight of the liver was also higher in the CAHF diet group than in the normal diet group (Fig. 3b). In contrast, the liver from the EPA diet group was not significantly different in appearance from that from the normal diet group, although it was still heavier than that from the control diet group $(\mathrm{p}<0.05)$. In addition, EPA administration was found not to improve liver injury assessed by measuring serum transaminase activity (Fig. 3c). The serum alanine aminotransferase (ALT) activity in the CAHF diet group was marginally higher than that in the normal diet group, although that in the EPA diet group was significantly higher than that in the normal diet group $(\mathrm{p}<0.05)$. The serum aspartate aminotransferase (AST) activity was significantly higher in the CAHF diet group than in the normal diet group, but that in the EPA diet group was much higher than that in the CAHF diet group $(p<0.05)$. As demonstrated by the liver histology, focal leukocyte infiltration appeared more frequently in the liver from the EPA diet group than in that from the CAHF diet group (Fig. 3d).
SOAT -2

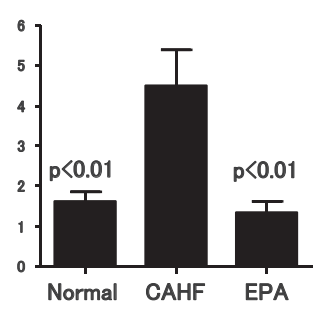

SCD-1

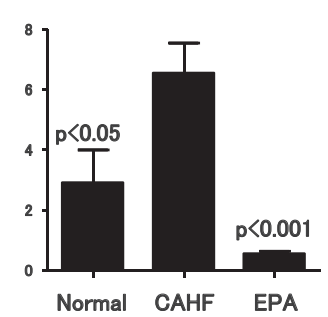

Cyp7a1

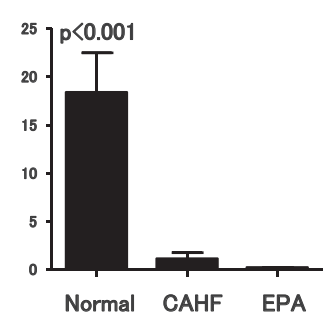

HMGCR

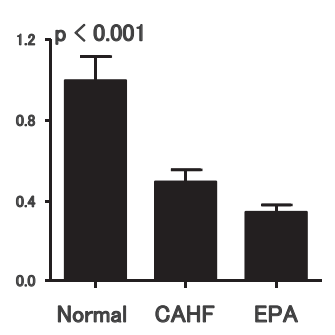

LDLR

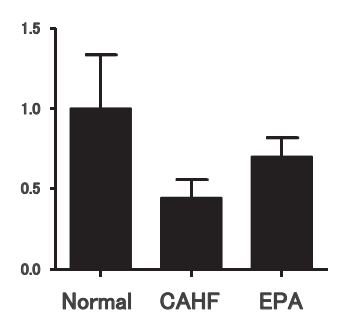

Fig. 2. Expression levels of mRNA encoding proteins related to regulation of the levels of cholesterol and cholesterol esters in liver. Real-time PCR was carried out using the specific primers listed in Table 4 to amplify the cDNA sequences obtained from mRNA of sterol $O$-acyltransferase-2 (SOAT-2), stearoyl-CoA desaturase-1 (SCD-1), cytochrome P-450 type 7a1 (Cyp7a1), 3-hydroxymethylglutaryl-CoA reductase (HMGCoAR) and low-density lipoprotein receptor (LDLR) mRNA. P values above the column and bar indicate statistically significant difference in the values compared with those of the CAHF diet group. 
a
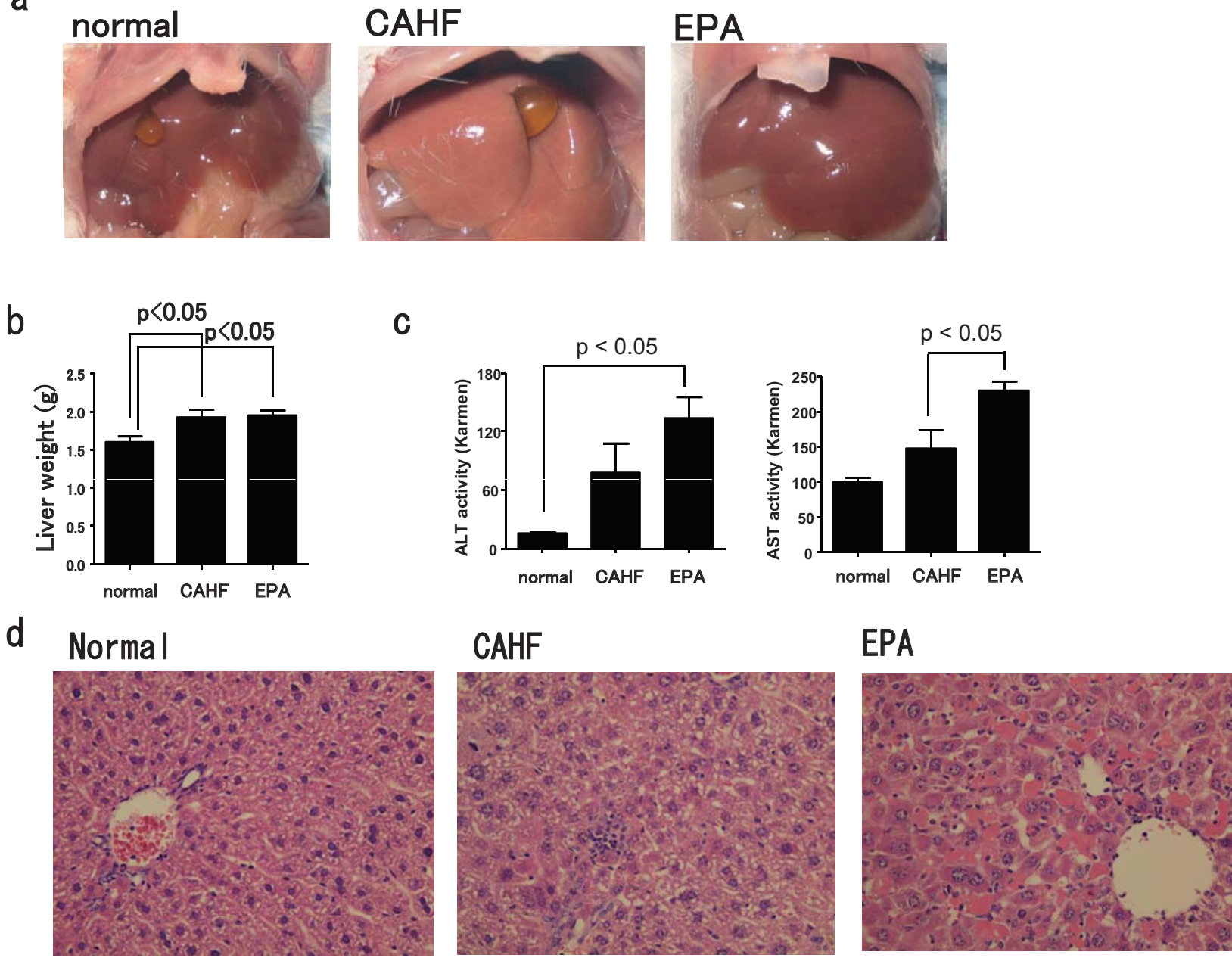

Fig. 3. The liver of the mice fed the CAHF diet was tinged with white and enlarged (a) compared with that of the mice fed the normal diet. However, the liver of the mice fed the EPA diet was similar in appearance to that of the mice fed the normal diet. The weight of the liver from the CAHF diet group increased compared with that from the normal diet $(p<0.001)(b)$. However, there was no difference in liver weight between the CAHF and EPA diet groups. Liver injury was compared by assessing the serum activities of alanine aminotransferase (ALT) and aspartate aminotransferase (AST) (c) and light microscopic observations of liver sections stained with hematoxylin-eosin (d).

\section{Expression levels of mRNA of inflammation- related proteins in liver}

To evaluate the extent of inflammatory responses occurring in the liver from the mice fed the test diet, the expression levels of the mRNA of cytokines, chemokines and proteins whose levels are predicted to change upon inflammation were determined. The expression levels of all the mRNA species shown in Fig. 4 did not significantly change in the liver from the CAHF diet group compared with that from the normal diet group. However, the expression levels of IL-1 $\beta$, CCL2, CCL5, ICAM-1 and serum amyloid 3 (Saa3) in the liver of the mice fed the EPA diet were significantly higher than those in the liver of the mice fed the CAHF diet.

\section{DISCUSSION}

The CAHF diet induced the concomitant elevation in the levels of SCD-1 and SOAT-2 mRNA in the liver of mice compared with the normal diet (Fig. 2), suggesting that the enhancement in the rates of MUFA generation from saturated fatty acids and of their esterification 
EPA aggravates experimental fatty liver injury

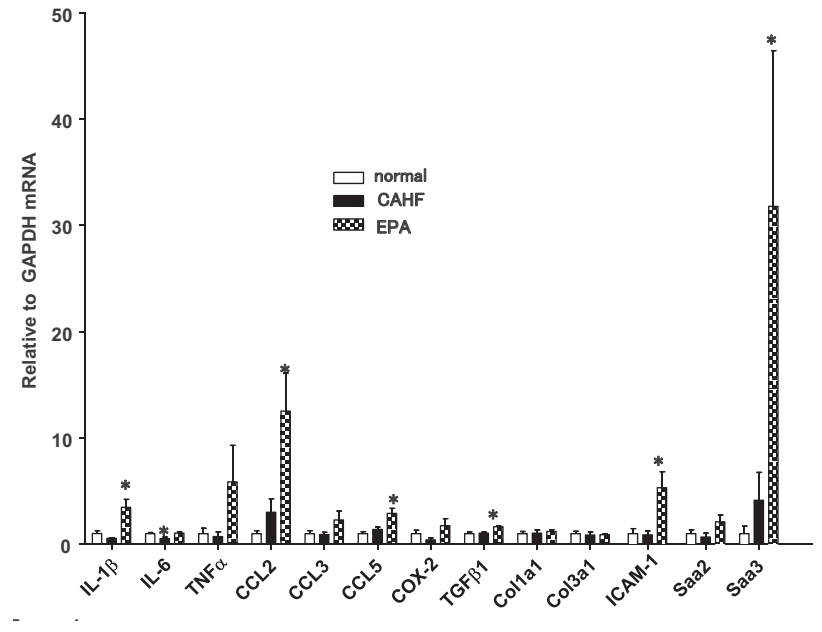

Fig. 4. Expression levels of mRNA of inflammation-related proteins in the liver. Real-time PCR was carried out using the specific primers listed in Table 3 to amplify the cDNA sequences obtained from mRNA shown in the figure. Statistically significant differences from the value of the CAHF diet group at $p$ of less than 0.05 are marked by an asterisk.

to cholesterol account for the selective accumulation of MUFA-containing CE in the liver (Table 5). There are several reports indicating that diets containing both cholesterol and cholate elevate MUFA level (Ueno and Okuyama, 1986) as well as SOAT activity in the liver (Erikson et al., 1980). In addition, the dietary administration of cholesterol alone induces the elevation in SCD enzymatic activity and the level of MUFA-containing CE in the liver (Landau et al., 1977). In contrast, there has so far been no report indicating the direct upregulation of SCD-1 or SOAT-2 expression in the liver by cholate supplementation. As has been shown in this study (Fig. 2), the reduction in Cyp7a1 mRNA level in the liver is a result of the action of cholic acid (Chiang et al., 2000). This effect hampers the mobilization of cholesterol into bile acid synthesis, which contributes to the elevation in cholesterol level. In addition, cholate can augment the intestinal absorption of dietary cholesterol (Woollett et al., 2004). Thus, we suppose that cholic acid in the CAHF diet indirectly contributes to the accumulation of MUFAcontaining $\mathrm{CE}$ in the liver through the action of cholesterol to enhance MUFA generation and MUFA esterification to cholesterol (Landau et al., 1977). We also note that MUFA such as oleic acid are preferentially esterified to cholesterol by SOAT-2 (Suckling and Stange, 1985). Therefore, enhanced MUFA synthesis from saturated fatty acids due to SCD-1 upregulation is related to the effi- cient synthesis of MUFA-containing CE by SOAT-2 in the liver of the mice fed the CAHF diet.

The suppression of SCD-1 expression by dietary EPA has already been demonstrated (Sekiya et al., 2003), however, it seems to be a common action of polyunsaturated fatty acids because arachidonic acid (20:4n-6) similarly suppresses SCD-1 expression (Mutch et al., 2005). These actions have been shown to be mediated through the downregulation of SREBP-1 expression (Sekiya et al., 2003; Yamazaki et al., 2007). In addition, it is reported that PUFA can suppress SOAT-2 expression in experimental animals (Botham et al., 2001; Lecker et al., 2011). Thus, the concomitant suppression of SCD-1 and SOAT-2 expression is considered to mediate the attenuation of the accumulation of MUFA-containing CE in the liver by EPA administration (Table 5). It has also been demonstrated that the genetic deletion of SCD-1 aggravates hepatic cell damage in the mouse methionine- and choline-deficient-diet-induced fatty liver model (Zheng et al., 2009). In addition, SCD-1 deficiency attenuates atherogenic lipid abnormalities but enhances inflammation and atherosclerosis (MacDonald et al., 2009). The role of LXR in SCD-1 induction in the prevention of saturatedfatty-acid-mediated lipotoxicity in endothelial injury was also reported (Peter et al., 2008). These studies suggest that SCD-1 plays a protective role in the pathogenesis of hepatic and vascular diseases through its ability to eliminate non-esterified saturated fatty acids (Gentile et al., 2008; Larter et al., 2008b). Recent studies suggested the potency of non-esterified free fatty acids to enhance bileacid-induced apoptosis (Pusl et al., 2008) and activate inflammatory responses in the liver (Csak et al., 2011). However, we could find no significant difference in the levels of saturated fatty acids and MUFA in the NEFA fraction in the liver of the mice fed the EPA diet compared with in the liver of those fed the CAHF diet (data not shown).

There are many reports indicating the preventive effects of EPA or EPA-rich fish oil in experimentally induced fatty liver (Hein et al., 2010; Jung et al., 2011; Kajikawa et al., 2009; Sekiya et al., 2003; Tanaka et al., 2010; Yamazaki et al., 2007). In addition, EPA administration could also ameliorate liver injury and fibrinogenesis occurring in experimental fatty liver models (Kajikawa et al., 2010; Svegliati-Baroni et al., 2006). Larter et al. (2008a) have shown that fish oil prevents fatty liver but not hepatic injury and enhances oxidative responses in the liver of mice fed a methinone- and choline-deficient diet. Our study demonstrated that EPA administration similarly attenuates fatty liver but aggravates liver injury and associated inflammatory responses (Figs. 3 and 4). As 
suggested by Larter et al. (2008a), the enhanced oxidative stress may be involved in the aggravation of liver injury by the EPA administration. The accumulated lipoperoxides are suggested to be proinflammatory and cause liver injury. The dietary levels of pro-oxidant in the EPA diet as well as the contents of lipoperoxides and their induction of stress responses in the liver of the mice fed the EPA diet should be further estimated. We also consider that EPA causes deleterious effects on fatty liver injury when CE synthesis predominantly occurs and plays a protective role as shown by the mice fed the CAHF diet. The effect of EPA or n-3-PUFA-rich fish oil has so far been examined using the fatty liver models associated with fatty liver due to TG accumulation (Hein et al., 2010; Jung et al., 2011; Kajikawa et al., 2009, 2010; Larter et al., 2008a; Sekiya et al., 2003; Svegliati-Baroni et al., 2006; Tanaka et al., 2010; Yamazaki et al., 2007). Further studies are necessary to define the relationship between the type of lipid accumulated in the liver and the effect of EPA or n-3-PUFA-rich fish oil on liver injury.

Note that EPA supplementation markedly reduces the arachidonic acid level (20:4n-6) and elevates the EPA level itself in the liver lipids (Table 5), suggesting that the generation of metabolites from these PUFA markedly changes in the liver. We speculate that the generation of 20:4n-6-derived metabolites contributing to the protection from inflammation and liver cell damages is inhibited in the liver of mice fed the EPA diet. We have already reported that dietary enrichment with $\alpha$-linolenic acid (18:3n-3), a metabolic precursor of EPA, elevates tissue EPA content and enhances acute hepatitis induced by the coadministration of lipopolysaccharide (LPS) and galactosamine (Watanabe and Okuyama, 1991). In addition, dietary $\alpha$-linolenic acid augments LPS-induced tumor necrosis factor $\alpha$ generation in mice (Watanabe et al., 1993), since endogenously generated $\mathrm{PGE}_{2}$ is an inhibitor of LPS-induced TNF production in macrophages (Kunkel et al., 1988). These results suggest that EPA administration enhances liver inflammation by enhancing TNF productivity upon treatment with an endotoxin. However, the expression level of TNF mRNA in the liver of mice fed the EPA diet was not significantly different from that in the liver of those fed the CAHF diet (Fig. 4). Recent studies have suggested that endotoxins play an important role in immunoinflammatory mechanisms in fatty liver development (Imajo et al., 2012; Tsujimoto et al., 2012). In addition, bile acids, which are possibly elevated in cholestasis as well as in the liver of mice fed the CAHF diet, are reported to directly induce hepatic inflammation (Allen et al., 2011). Therefore, there are possibilities that cholate added in the CAHF diet might act as an agonist to elicit transcriptional responses leading to inflammation in liver and that EPA may aggravate liver inflammation by interacting more directly with cholate. For more detailed analysis of the interaction EPA with endotoxins as well as cholate in enhancing inflammatory responses, the comparison of the data from dietary group of mice fed the EPAsupplemented normal diet should be carried out. Furthermore, the diets used in this study were not balanced in their total energy and macronutrients (Table 2). In particular, protein contents were much lower in the CAHF and EPA diets than in the normal diet (Table 2). These diets might create differential nutritional statuses of the mice at 5 weeks of age, which are still in the intense growth phases, even though their energy intake and body weight gain were quite similar (Fig. 1). The use of iso-caloric/ semipurified diets should be considered in future studies.

In conclusion, EPA supplementation attenuates $\mathrm{CE}$ accumulation in the liver of mice fed the CAHF diet but aggravates liver injury and inflammation in such mice. The role of oxidative stress as well as arachidonic acid metabolism in the aggravation of liver injury by EPA administration still needs to be investigated.

\section{ACKNOWLEDGMENT}

The authors thank Mr.Yasuhiko Yoshida for his excellent technical assistance in the biochemical assays and fatty acid analysis. This work was supported in part by a Grant-in-Aid from the Ministry of Education, Culture, Sports, Science and Technology of Japan (Research Project Number: 23590873)

\section{REFERENCES}

Allen, K., Jaeschke, H. and Copple, B.L. (2011): Bile acids induce inflammatory genes in hepatocytes: A novel mechanism of inflammation during obstructive cholestasis. Am. J. Pathol., 178, $175-186$.

Bligh, E.G. and Dyer, W.J. (1959): A rapid method of total lipid extraction and purification. Can. J. Biochem. Physiol., 37, 911917.

Botham, K.M., Maldonado, E.N., Chico, Y., Zheng, X., Avella, M. and Ochoa, B. (2001): The influence of chylomicron remnants on cholesteryl ester metabolism in cultured rat hepatocytes: comparison of the effects of particles enriched in n-3 or n-6 polyunsaturated fatty acids. Biochim. Biophys. Acta, 1534, 96-109.

Chen, W., Suruga, K., Nishimura, N., Gouda, T., Lam, V.N. and Yokogoshi, H. (2005): Comparative regulation of major enzymes in the bile acid biosynthesis pathway by cholesterol, cholate and taurine in mice and rats. Life Sci., 77, 746-757.

Chiang, J.Y., Kimmel, R., Weinberger, C. and Stroup, D. (2000): Farnesoid $\mathrm{X}$ receptor responds to bile acids and represses cholesterol 7alpha-hydroxylase gene (CYP7A1) transcription. J. Biol. Chem., 275, 10918-10924.

Csak, T., Ganz, M., Pespisa, J., Kodys, K., Dolganiuc, A. and 
EPA aggravates experimental fatty liver injury

Szabo, G. (2011): Fatty acid and endotoxin activate inflammasomes in mouse hepatocytes that release danger signals to stimulate immune cells. Hepatology, 54, 133-144.

Erickson, S.K., Shrewsbury, M.A., Brooks, C. and Meyer, D.J. (1980): Rat liver acyl-coenzyme A: cholesterol acyltransferase: its regulation in vivo and some of its properties in vitro. J. Lipid Res., 21, 930-941.

Gentile, C.L. and Pagliassptti, M.J. (2008): The role of fatty acids in the development and progression of nonalcoholic fatty liver disease, J. Nutr. Biochem., 19, 567-576.

Hara, A. and Radin, N.S. (1978): Lipid extraction of tissues with a low-toxicity solvent. Anal. Biochem., 90, 420-426.

Hein, G.J., Bernasconi, A.M., Montanaro, M.A., Pellon-Maison, M., Finarelli, G., Chicco, A., Lombardo, Y.B. and Brenner, R.R. (2010): Nuclear receptors and hepatic lipidogenic enzyme response to a dyslipidemic sucrose-rich diet and its reversal by fish oil n-3 polyunsaturated fatty acids. Am. J. Physiol. Endocrinol. Metabol., 298, E429-439.

Heuman, D.M. (1989): Quantitative estimation of the hydrophilichydrophobic balance of mixed bile salt solutions. J. Lipid. Res., 30, 719-730.

Imajo, K., Fujita, K., Yoneda, M., Nozaki, Y., Ogawa, Y., Shinohara, Y., Kato, S., Mawatari, H., Shibata, W., Kitani, H., Ikejima, K., Kirikoshi, H., Nakajima, N., Saito, S., Maeyama, S., Watanabe, S., Wada, K. and Nakajima, A. (2012): Hyperresponsivity to low-dose endotoxin during progression to nonalcoholic steatohepatitis is regulated by leptin-mediated signaling. Cell Metab., 16, 44-54.

Jung, U.J., Millman, P.N., Tall, A.R. and Deckelbaum, R.J. (2011): n-3 Fatty acids ameliorate hepatic steatosis and dysfunction after LXR agonist ingestion in mice. Biochim. Biophys. Acta., 1811, 491-497.

Kajikawa, S., Harada, T., Kawashima, A., Imada, K. and Mizuguchi, K. (2009): Highly purified eicosapentaenoic acid prevents the progression of hepatic steatosis by repressing monounsaturated fatty acid synthesis in high-fat/high-sucrose diet-fed mice. Prostaglandins Leukot. Essent. Fatty Acids, 80, 229-238.

Kajikawa, S., Harada, T., Kawashima, A., Imada, K. and Mizuguchi, K. (2010): Highly purified eicosapentaenoic acid ethyl ester prevents development of steatosis and hepatic fibrosis in rats. Dig. Dis. Sci., 55, 631-641.

Keitel, V., Kubitz, R. and Häussinger, D. (2008): Endocrine and paracrine role of bile acids. World J. Gastroenterol., 14, 56205629.

Kunkel, S.L., Spengler, M., May, M.A., Spengler, R., Larrick, J. and Remick, D. (1988): Prostaglandin $\mathrm{E}_{2}$ regulates macrophagederived tumor necrosis factor gene expression. J. Biol. Chem., 263, 5380-5384.

Landau, J.M., Sekowski, A. and Hamm, M.W. (1977): Dietary cholesterol and the activity of stearoyl CoA desaturase in rats: evidence for an indirect regulatory effect. Biochim. Biophys. Acta., 1345, 349-357.

Larter, C.Z., Yeh, M.M., Cheng, J., Williams, J., Brown, S., de la Pena, A., Bell-Anderson, K.S. and Farrell, G.C. (2008a): Activation of peroxisome proliferator-activated receptor alpha by dietary fish oil attenuates steatosis, but does not prevent experimental steatohepatitis because of hepatic lipoperoxide accumulation. J. Gastroenterol. Hepatol., 23, 267-275.

Larter, C.Z., Yeh, M.M., Haigh, W.G., Williams, J., Brown, S., Bell-Anderson, K.S., Lee, S.P. and Farrell, G.C. (2008b): Hepatic free fatty acids accumulate in experimental steatohepatitis: role of adaptive pathways. J. Hepatol., 48, 638-647.
Lecker, J.L., Matthan, N.R., Billheimer, J.T., Rader, D.J. and Lichtenstein, A.H. (2011): Changes in cholesterol homeostasis modify the response of F1B hamsters to dietary very long chain n-3 and n-6 polyunsaturated fatty acids. Lipids Health Dis., 10, 186.

Lefebvre, P., Cariou, B., Lien, F., Kuipers, F. and Staels, B. (2009): Role of bile acids and bile acid receptors in metabolic regulation. Physiol. Rev., 89, 147-191.

MacDonald, M,L., van Eck, M., Hildebrand, R.B., Wong, B.W., Bissada, N., Ruddle, P., Kontush, A., Hussein, H., Pouladi, M.A., Chapman, M.J., Fievet, C., van Berkel, T.J., Staels, B., McManus, B.M. and Hayden, M.R. (2009): Despite antiatherogenic metabolic characteristics, SCD1-deficient mice have increased inflammation and atherosclerosis. Arterioscler. Thromb. Vasc. Biol., 29, 341-347.

Mutch, D.M., Grigorov, M., Berger, A., Fay, L.B., Roberts, M.A., Watkins, S.M., Williamson, G. and German, J.B. (2005): An integrative metabolism approach identifies stearoyl-CoA desaturase as a target for an arachidonate-enriched diet. FASEB J., 19, 599-601.

Peter, A., Weigert, C., Staiger, H., Rittig, K., Cegan, A., Lutz, P., Machicao, F., Häring, H.U. and Schleicher, E. (2008): Induction of stearoyl-CoA desaturase protects human arterial endothelial cells against lipotoxicity. Am. J. Physiol. Endocrinol. Metab., 295, E339-349.

Pusl, T., Wild, N., Vennegeerts, T., Wimmer, R., Göke, B., Brand, S. and Rust, C. (2008): Free fatty acids sensitize hepatocytes to bile acid-induced apoptosis. Biochem. Biophys. Res. Commun., 371, 441-445.

Rost, D., Herrmann, T., Sauer, P., Schmidts, H-L., Stieger, B., Meier, P.J., Stremmel, W. and Stiehl, A. (2003): Regulation of rat organic anion transporters in bile salt-induced cholestatic hepatitis: Effect of ursodeoxycholate. Hepatology, 38, 187-195.

Sekiya, M., Yahagi, N., Matsuzaka, T., Najima, Y., Nakakuki, M., Nagai, R., Ishibashi, S., Osuga, J., Yamada, N. and Shimano, H. (2003): Polyunsaturated fatty acids ameliorate hepatic steatosis in obese mice by SREBP-1 suppression. Hepatology, 38, 15291539.

Song, P., Zhang, Y. and Klaassen, C.D. (2011): Dose-response of five bile acids on serum and liver bile acid concentrations and hepatotoxicity in mice. Toxicol. Sci., 123, 359-367.

Suckling, K.E. and Stange, E.F. (1985): Role of acyl-CoA: cholesterol acyltransferase in cellular cholesterol metabolism. J. Lipid. Res., 26, 647-671.

Sugiyama, E., Ishikawa, Y., Li, Y., Kagai, T., Nobayashi, M., Tanaka, N., Kamijo, Y., Yokoyama, S., Hara, A. and Aoyama, T. (2008): Eicosapentaenoic acid lowers plasma and liver cholesterol levels in the presence of peroxisome proliferators-activated receptor alpha. Life Sci., 83,19-28.

Svegliati-Baroni, G., Candelaresi, C., Saccomanno, S., Ferretti, G., Bachetti, T., Marzioni, M., De Minicis, S., Nobili, L., Salzano, R., Omenetti, A., Pacetti, D., Sigmund, S., Benedetti, A. and Casini, A. (2006): A model of insulin resistance and nonalcoholic steatohepatitis in rats: role of peroxisome proliferator-activated receptor-alpha and n-3 polyunsaturated fatty acid treatment on liver injury. Am. J. Pathol., 169, 846-860.

Tanaka, N., Zhang, X., Sugiyama, E., Kono, H., Horiuchi, A., Nakajima, T., Kanbe, H., Tanaka, E., Gonzalez, F.J. and Aoyama, T. (2010): Eicosapentaenoic acid improves hepatic steatosis independent of PPAR $\alpha$ activation through inhibition of SREBP-1 maturation in mice. Biochem. Pharmacol., 80, 16011612. 


\section{S. Watanabe and K. Tsuneyama}

Teng, S. and Piquette-Miller, M. (2007): Hepatoprotective role of PXR activation and MRP3 in cholic acid-induced cholestasis. Br. J. Pharmacol., 151, 367-376.

Trauner, M., Baghdasaryan, A., Caludel, T., Ficket, P., Halibasic, E., Moustafa, T. and Zollner, G. (2011): Targeting nuclear bile acid receptors for liver diseases. Dig. Dis., 29, 98-102.

Tsujimoto, T., Kawaratani, H., Kitazawa, T., Uemura, M. and Fukui, H. (2012): Innate immune reactivity of the ileum-liver axis in nonalcoholic steatohepatitis. Dig. Dis. Sci., 57, 1144-1151.

Uchida, K., Nomura, Y. and Takeuchi, N. (1980): Effects of cholic acid, chenodeoxycholic acid, and their related bile acids on cholesterol, phospholipid, and bile acid levels in serum, liver, bile, and feces of rats. J. Biochem., 87, 187-194.

Ueno, K. and Okuyama, H. (1986) A high cholesterol/cholate diet induced fatty liver in spontaneous hypertensive rats. Lipids, 21, 475-480.

Vergnes, L. and Phan, J. (2003): Cholesterol and cholate components of an atherogenic diet induced distinct stages of hepatic inflammation. J. Biol. Chem., 278, 42774-42784.

Wang, L., Han, Y., Kim, C.S., Lee, Y.K. and Moore, D.D. (2003): Resistance of SHP-null mice to bile acid-induced liver damage. J. Biol. Chem., 278, 44475-44481.

Watanabe, M., Houten, S.M., Wang, L., Moschetta, A., Mangelsdorf, D.J., Heyman, R.A., Moore, D.D. and Auwerx, J. (2004): Bile acids lower triglyceride levels via a pathway involving FXR, SHP, and SREBP-1c. J. Clin. Invest., 113, 1408-1418.
Watanabe, S. and Okuyama, H. (1991): Effect of dietary alpha-linolenate/linoleate balance on endotoxin-induced hepatitis in mice. Lipids, 26, 467-471.

Watanabe, S., Onozaki, K., Yamamoto, S. and Okuyama, H. (1993): Regulation by dietary essential fatty acid balance of tumor necrosis factor production in mouse macrophages. J. Leukoc. Biol., 53, 151-156.

Watanabe, S. and Tsuneyama, K. (2010): A triglyceride-lowering effect of cattle bile is associated with elevation of cholesterol levels and liver injury in mice. J. Trad. Med., 27, 179-185.

Watanabe, S. and Tsuneyama, K. (2012): Cattle bile but not bear bile or pig bile induce lipid profile changes and fatty liver injury in mice. J. Toxicol. Sci., 37, 105-121.

Woollett, L.A., Buckley, D.D., Yao, L., Jones, P.J., Granholm, N.A., Tolley, E.A., Tso, P. and Heubi, J.E. (2004): Cholic acid supplementation enhances cholesterol absorption in humans. Gastroenterology, 126, 724-731.

Yamazaki, T., Nakamori, A., Sasaki, E., Wada, S. and Ezaki, O. (2007): Fish oil prevents sucrose-induced fatty liver but exacerbates high-safflower oil-induced fatty liver in ddY mice. Hepatology, 46, 1779-1790.

Zheng, Z., Berk, M., McIntyre, T.M. and Feldstein, A.E. (2009): Hepatic lipid partitioning and liver damage in nonalcoholic fatty liver disease: role of stearoyl-CoA. J. Biol. Chem., 284, 56375644. 